\title{
Pensamento Computacional sob a visão dos profissionais da computação: uma discussão sobre conceitos e habilidades
}

\author{
Ana Liz Souto Oliveira de Araújo ${ }^{12}$, Wilkerson L. Andrade ${ }^{1}$, \\ Dalton D. Serey Guerrero ${ }^{1}$
}

${ }^{1}$ Laboratório de Práticas de Software - Universidade Federal de Campina Grande Av. Aprígio Veloso, s/n, SPLab, Bodocongó

58429-900 - Campina Grande - PB - Brasil

${ }^{2}$ Departamento de Ciências Exatas - Universidade Federal da Paraíba

Rua da Mangueira, s/n, Companhia de Tecidos Rio Tinto

58.297-000 - Rio Tinto - PB - Brasil

analiz@copin.ufcg.edu.br, \{wilkerson, dalton\}@computacao.ufcg.edu.br

\begin{abstract}
Computational Thinking (CT) is an approach to solve problems based on Computer Science concepts. The heart of the question lies in the mental process involved in how computer professionals act when put into action practices and techniques of computer science to solve problems. We conducted a survey with computer professionals in order to find out their understanding about CT. Our results show that $64 \%$ are unaware of CT, and they suggest that the term and relevant skills are unknown by professionals of both industry and academia.
\end{abstract}

Resumo. Pensamento Computacional (PC) consiste em uma abordagem de resolução de problemas que explora conceitos da computação. $O$ cerne da questão encontra-se nos processos mentais envolvidos na maneira como um profissional da computação age quando coloca em ação técnicas e práticas da ciência da computação para solucionar problemas. Neste trabalho, realizamos um survey com profissionais da computação para capturar a compreensão que eles têm de PC. Nossos resultados apontam que 64\% dos pesquisados desconhecem o PC e sugerem que o termo e habilidades relevantes são pouco conhecidos e compreendidos pelos profissionais, tanto na indústria como na academia.

\section{Introdução}

Pensamento Computacional (PC) foi apresentado em 2006 por Jeannette Wing associado às ideias de resolução de problemas, design de sistemas e compreensão do comportamento humano norteados por conceitos fundamentais da Ciência da Computação (Wing, 2006). Foi difundido que se trata de um conjunto de conceitos, habilidades e práticas da computação que podem ser aplicados tanto em atividades do cotidiano como em outras áreas do conhecimento. A importância do PC foi comparada às competências de ler, escrever e calcular.

A comunidade de Educação em Computação acolheu a ideia inicial e nos 


\section{CBIE-LACLO 2015}

Anais dos Workshops do IV Congresso Brasileiro de Informática na Educação (CBIE 2015)

últimos anos inúmeros trabalhos foram publicados. Esses versam sobre propostas de atividades, relatos de experiências e pesquisas empíricas sobre adoção e avaliação do pensamento computacional nos diversos níveis de ensino (Mannila et al, 2014) (Walden et al, 2013) (Barr e Stephenson, 2009). Outros investigam a respeito da definição formal para o PC (Selby e Woollard, 2014)(Hu, 2011).

A definição adotada neste trabalho é que PC consiste em uma abordagem de resolução de problemas incorporando processos mentais e ferramentas que utilizam habilidades como organização e análise de dados, construção de algoritmos, abstração, criação de modelos, simulação, automatização de soluções e paralelização. Essa lista de habilidades auxilia a definir um escopo sobre o que se espera de uma abordagem de resolução de problemas no contexto do PC. Essa definição foi escolhida por ser a definição operacional adotada pela International Society for Technology in Education (ISTE) e Computer Science Teachers Association (CSTA).

Por essa definição, o cerne do escopo da resolução de problemas encontra-se nos processos mentais envolvidos na maneira como um profissional da computação age quando coloca em ação técnicas e práticas da ciência da computação para solucionar problemas. Assim, espera-se que profissionais da computação, por terem, geralmente, essas habilidades desenvolvidas durante sua formação acadêmica, reconheçam e explorem essas habilidades para a resolução de problemas tanto no ambiente de trabalho como em atividades do cotidiano.

Nesse contexto, nós levantamos as seguintes questões de pesquisa: QP1 - Qual a percepção do termo Pensamento Computacional para os profissionais da computação?; QP2 - Existe diferença significativa percepção do PC quando comparados os profissionais da academia (professores, estudantes de pós-graduação e pesquisadores) aos profissionais da indústria (desenvolvedores, analistas, engenheiros de software)?; QP3 - Os profissionais da computação reconhecem que exploram habilidades associadas ao PC nas suas atividades laborais? QP4 - Os profissionais da computação reconhecem que exploram habilidades associadas ao PC em suas atividades do cotidiano, fora do seu ambiente de trabalho?

O objetivo do nosso trabalho é capturar a percepção de PC e de habilidades associadas sob a ótica do profissional da computação. Consideramos profissionais da computação pessoas graduadas em cursos de computação (bacharelados e licenciaturas). Nosso método de pesquisa empregado foi o survey exploratório e o instrumento de coleta de dados foi um questionário online. Nós selecionamos a amostra por meio do método não-probabilístico snowball sampling.

A contribuição do nosso trabalho é levantar a discussão sobre a percepção que os profissionais da computação possuem a respeito do PC e das habilidades associadas. Nossos resultados sugerem que o termo e as habilidades relevantes são pouco conhecidos e compreendidos pelos profissionais, tanto na indústria como na academia.

O restante do trabalho está organizado da seguinte maneira: na Seção 2 apresentamos os conceitos e habilidades associadas ao PC; na Seção 3 listamos trabalhos relacionados; na Seção 4 descrevemos a metodologia; na Seção 5 discutimos os resultados; e na Seção 6 realizamos as considerações finais do trabalho. 


\section{Pensamento Computacional: resolução de problemas explorando habilidades}

Pensamento Computacional é uma abordagem focada na resolução de problemas explorando processos cognitivos, técnicas e ferramentas comuns na Ciência da Computação. Embora o termo faça uma alusão direta à Computação, nem todos os processos e técnicas associados são exclusivos da Computação. Mesmo assim, eles foram incorporados e são amplamente explorados no contexto de buscar soluções mais eficientes para resolver problemas ou atividades complexas.

Definições mais amplas afirmam que PC consiste em resolver problemas, automatizar sistemas ou transformar dados através da construção de modelos $\mathrm{e}$ representações, concretas ou abstratas $(\mathrm{Hu}, 2011)$. Outras argumentam que PC é uma atividade que possui orientação processual e formal, enfatizando processos e estratégias para resolução de problemas (Walden et al, 2013). Já outra afirma que PC é um termo que engloba um conjunto de conceitos e processos da Ciência da Computação, os quais auxiliam na formulação de soluções em diferentes campos do saber (Mannila et al, 2014).

As habilidades associadas ao PC variam conforme se investiga na literatura. Abstração, decomposição e construção de algoritmos são habilidades em que há consenso de significação e aparecem com maior frequência (Selby e Woollard, 2014) (Mannila et al, 2014) (Barr e Stephenson, 2009). Abstração consiste na ideia de identificar o essencial do que se está observando. Pode-se ainda criar várias camadas de abstração sobre a mesma observação, onde cada camada considera um nível de complexidade de detalhes.

Decomposição é a capacidade de lidar com problemas complexos, dividindo-o em tarefas menores e mais fáceis de gerenciar e resolver. Construção de algoritmos é a competência de planejar e organizar uma sequência de passos para resolver um problema ou alcançar um objetivo (Selby e Woollard, 2014).

Avaliação e generalização são outras duas habilidades destacadas (Selby e Woollard, 2014). Avaliação é interpretada como a comparação entre opções com relação à análise de recursos, sejam de tempo, espaço, esforço ou tamanho, e a escolha da melhor opção baseada nesses recursos. Generalização consiste em partir de algo específico para uma aplicação mais ampla, ou seja, reconhecer padrões comuns, que compartilham características comuns, e aplicar em outros contextos. Na resolução de problemas, generalização significa identificar partes funcionais que podem ser usadas ou recombinadas na resolução de problemas similares.

No contexto da educação norte-americana, nove habilidades são listadas como núcleo do PC (Barr e Stephenson, 2009). Essas habilidades estão em conformidade com CSTA e ISTE. Além de abstração, decomposição e criação de algoritmos, consideramse também coleta, análise e representação de dados, simulação, automação e paralelização. Simulação diz respeito a usar ou criar simulações para execução de testes e experimentos. Automação significa reconhecer como a tecnologia pode nos ajudar a realizar tarefas que de outra maneira seriam repetitivas, inviáveis ou difíceis de realizar. E paralelização consiste em organizar recursos para realizar tarefas simultâneas. 


\section{Trabalhos relacionados}

Em (Mannila et al, 2014) foi realizado um survey com professores da educação básica dos Estados Unidos e países da Europa no intuito de revelar em que medida aspectos do PC já fazem parte da prática dos professores em sala de aula. Os resultados sugerem que alguns professores já estão envolvidos em atividades que têm um forte potencial para a introdução de alguns aspectos do PC.

Em (Pokorny e White, 2012) foi apresentado o relato de um workshop para professores da educação básica americana, no intuito de apresentar tecnologias atuais para uso em sala de aula. O survey realizado apontou que os professores possuíam conhecimentos limitados a respeito da computação e que nenhum participante era familiarizado com o conceito do PC anterior ao workshop.

Em (Li e Wang, 2012) é apresentado o resultado da experiência de dois anos de um curso de introdução ao PC para alunos sem conhecimento prévio em computação. Os alunos relataram no survey que necessitam de conceitos teóricos mínimos sobre Ciência da Computação para entender o PC.

Nosso trabalho difere dos demais pois os sujeitos do estudo são profissionais da computação. Nossa discussão foca-se na percepção que eles já possuem a respeito do PC. Os trabalhos encontrados na literatura buscam disseminar o PC por meio de cursos, geralmente direcionados a professores e alunos da educação básica, e medir a consciência deles sobre PC após participação no curso.

\section{Metodologia}

O método de pesquisa empregado no nosso trabalho foi um survey exploratório não supervisionado. Utilizamos como instrumento de coleta de dados um questionário online com oito questões objetivas e uma subjetiva. Adaptamos questões do questionário aplicado em (Mannila et al, 2014) para professores da educação básica. Realizamos a avaliação do questionário por meio de um teste piloto com um subconjunto da amostra e descartamos as respostas.

Nosso questionário foi planejado, em sua primeira parte, para capturar a visão pessoal sobre o termo PC dos respondentes, com a pergunta discursiva "Qual seu entendimento sobre Pensamento Computacional?". E assim, responder as questões de pesquisa 1 e 2 :

QP1 - Qual a percepção do termo Pensamento Computacional para os profissionais da computação?

QP2 - Há diferença significativa na proporção de respostas convergentes para a definição do termo Pensamento Computacional quando comparados os profissionais da academia aos profissionais da indústria?

Já na segunda parte do questionário, planejamos capturar a percepção de habilidades associadas ao PC em duas situações distintas: nas atividades laborais e nas atividades cotidianas. E dessa forma, responder as questões de pesquisa 3 e 4 :

QP3 - Os profissionais da computação reconhecem que exploram habilidades associadas ao PC nas suas atividades laborais? 
QP4 - Os profissionais da computação reconhecem que exploram habilidades associadas ao PC nas suas atividades cotidianas, fora do ambiente de trabalho?

Nós selecionamos as nove habilidades descritas na definição da CSTA: coleta de dados, análise de dados, representação de dados, algoritmo, decomposição, abstração, automação, simulação e paralelização. No questionário, as habilidades foram descritas, conforme Quadro 1, e solicitamos que as pessoas classificassem cada uma delas em uma escala likert com as opções: (1) nunca exploro, (2) exploro raramente, (3) exploro às vezes, (4) exploro frequentemente e (5) exploro sempre. Essa classificação foi solicitada primeiro relacionada às atividades laborais e em seguida, relacionada às atividades do cotidiano, sendo essa última exibida no Quadro 1. Na terceira parte do questionário, nós solicitamos informações relacionadas a sexo, formação acadêmica, tempo e área de atuação.

Quadro 1 - Descrição das habilidades do PC no questionário

4. No seu cotidiano, fora do seu ambiente de trabalho, quais dos itens abaixo descrevem habilidades que você explora em suas atividades do dia a dia?

- Coleta informações adequadas e seleciona informações relevantes para o contexto da atividade desenvolvida (coleta de dados).

- Dá sentido aos dados, encontra padrões e obtêm conclusões a partir da observação dos dados (análise de dados)

- Organiza e descreve dados em representações apropriadas, como gráficos, palavras, frases, imagens, tabelas, etc. (representação de dados).

- Divide tarefas grandes em partes menores e mais fáceis de gerenciar e resolver (decomposição de problemas).

- Planeja e organiza sequências de passos para resolver um problema ou alcançar um objetivo (algoritmos). - Reduz a complexidade para definir a ideia principal, encontra características básicas e cria modelos (abstração)

- Usa ou cria simulações, por exemplo, para a execução de testes e experimentos (simulação).

- Reconhece como a tecnologia pode nos ajudar a realizar novas tarefas que de outra maneira seria repetitiva, inviável ou difícil de realizar (automação).

Organiza recursos para, simultaneamente e de forma cooperativa, realizar tarefas para alcançar um objetivo (paralelização).

A população da pesquisa abrangeu profissionais formados em cursos de computação (bacharelados e licenciaturas). Optamos por uniformizar os profissionais graduados nesses cursos chamando-os de "profissionais da computação". Selecionamos a amostra por meio do método não-probabilístico snowball sampling. Enviamos e-mails com o questionário inicialmente para pessoas próximas e expandimos para as indicações reportadas.

No total, enviamos 124 e-mails solicitando participação na pesquisa. Desses, 58 questionários foram respondidos. A taxa de retorno foi de aproximadamente $46 \%$. A formação acadêmica dos participantes foi: sistema de informação, análise e desenvolvimento de sistemas, processamento de dados, ciência da computação (licenciatura e bacharelado). Os participantes residem principalmente nos estados da Paraíba e do Rio Grande do Norte.

\section{Resultados e discussões}

Discutimos os resultados em duas partes: análise da definição do termo PC e análise das habilidades associadas ao PC.

\subsection{Análise da definição do termo "Pensamento Computacional"}

Examinamos as respostas da questão discursiva "Qual seu entendimento sobre Pensamento Computacional?" no intuito de responder a questão de pesquisa 1: 


\section{CBIE-LACLO 2015}

Anais dos Workshops do IV Congresso Brasileiro de Informática na Educação (CBIE 2015)

\section{QP1 - Qual a percepção do termo PC para os profissionais da computação?}

Classificamos as respostas de acordo com a definição da CSTA adotada neste trabalho: PC consiste em uma abordagem de resolução de problemas incorporando processos mentais e ferramentas que utilizam habilidades como organização e análise de dados, construção de algoritmos, abstração, decomposição, simulação, automatização e paralelização.

As 58 respostas foram classificadas em dois grupos: grupo A - respostas que faziam alusão a resolução de problemas (21 respostas) e grupo B - respostas equivocadas ou que afirmaram não deter conhecimento (37 respostas). De acordo com a classificação, 36\% dos profissionais (21 de 58) descreveram que PC é um método de resolução de problemas. Já 64\% dos profissionais (37 de 58) desconhecem o termo PC.

Nós tentamos fazer outra classificação considerando as habilidades relatadas nas respostas do grupo A. Entretanto, a única habilidade citada foi algoritmo em apenas 5 das 21. As demais respostas apenas mencionavam que se tratava de habilidades comuns na computação, sem especificação. Nenhuma resposta citou duas ou mais competências.

Esses resultados sugerem que os profissionais da computação possuem pouco entendimento a respeito do PC e das competências associadas. Nós percebemos que a classificação das respostas baseada na concordância com a definição adotada se restringiu a classificação binária entre conhecer ou não o PC como um método de resolução de problemas, sem necessariamente relacionar competências. O resultado obtido era esperado, uma vez que o PC é um termo que ganhou destaque a partir de 2006. Ademais, supomos ser um assunto de interesse geralmente restrito a um subgrupo da área de Educação em Computação.

Em seguida, buscamos investigar se a percepção do conceito de PC estava associada a área de atuação profissional. Assim, dividimos os profissionais em dois grupos: i) Academia corresponde às pessoas que responderam que atuam como professores, estudantes de pós-graduação e pesquisadores e ii) indústria corresponde às pessoas que responderam que atuam como desenvolvedores, analistas de sistemas, analistas de testes, engenheiros de software. A Tabela 1 mostra esses resultados.

Tabela 1: Tabela de contingência

\begin{tabular}{|l|l|l|l|}
\hline & Grupo A & Grupo B & Total \\
\hline Academia & $10(40 \%)$ & $15(60 \%)$ & 25 \\
\hline Indústria & $11(33 \%)$ & $22(67 \%)$ & 33 \\
\hline
\end{tabular}

Após a divisão, retornamos à questão de pesquisa QP2, seguida das hipóteses para verificar se há diferença significativa no percentual das respostas:

QP2 - Há diferença significativa na proporção de respostas convergentes para a definição do termo PC quando comparados os profissionais da academia aos profissionais da indústria?

H1-0: Não há diferença entre a proporção de respostas convergentes para a definição do termo PC quando comparados os profissionais da academia aos profissionais da indústria. 


\section{CBIE-LACLO 2015}

Anais dos Workshops do IV Congresso Brasileiro de Informática na Educação (CBIE 2015)

H1-1: Há diferença entre a proporção de respostas convergentes para a definição do termo PC quando comparados os profissionais da academia aos profissionais da indústria.

Testamos a hipótese usando teste de proporção com nível de confiança $95 \%$. O p-value obtido foi 0.8047 e para esse valor não podemos rejeitar a hipótese nula H1-0. O intervalo de confiança calculado está entre $-20 \%$ e $35 \%$. Para esse intervalo, o valor zero é um valor plausível para as diferenças nos percentuais, e portanto não temos evidências estatísticas de que o percentual entre os grupos diferem.

Nossos resultados sugerem que independente da área de atuação, o termo PC é pouco compreendido. Também não podemos supor que os profissionais da academia possuem mais ciência do termo PC quando comparados aos profissionais da indústria. Nossa expectativa inicial era de que a diferença entre academia e indústria fosse maior, pois os profissionais da academia estão mais atentos e abertos às discussões de novos assuntos da literatura.

\subsection{Análise das habilidades associadas ao Pensamento Computacional}

Analisamos as respostas da segunda parte do questionário as quais capturavam a percepção de habilidades do PC em duas situações distintas: em atividades laborais e em atividades cotidianas. A Figura 1 mostra os gráficos resultantes.
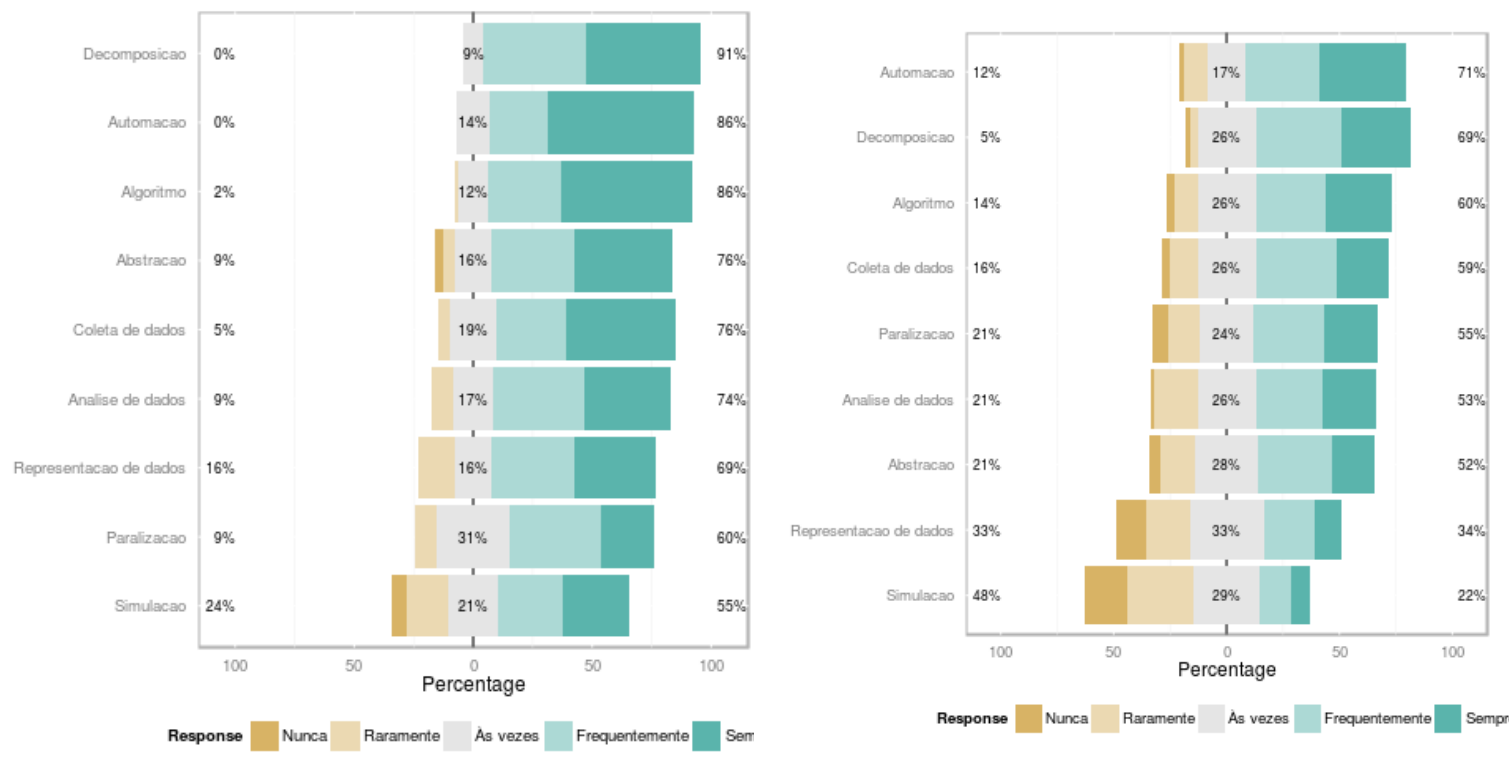

Figura 1: Percepção das habilidades do PC em atividades laborais e cotidianas, respectivamente.

Nesse contexto, retornamos a questão de pesquisa 3: QP3- Os profissionais da computação reconhecem que exploram habilidades associadas ao PC nas suas atividades laborais?

Para cada habilidade, realizamos um teste de proporção no intuito de analisar o intervalo de confiança das proporções entre as respostas que afirmaram explorar frequentemente ou sempre e as respostas que afirmaram explorar às vezes, raramente ou nunca a habilidade em questão. Calculamos o teste de proporção com nível de confiança 
de $95 \%$ para cada habilidade e sumarizamos os resultados na Tabela 2.

Nossa análise irá se deter apenas nos intervalos de confiança. Observamos os resultados calculados na Tabela 2 e vemos que as habilidades de coleta de dados, análise de dados, representação de dados, decomposição, algoritmo, abstração e automação apresentam intervalos de confiança sempre acima de 56\%. Isso significa que, se executarmos esse experimento 100 vezes, esse resultado deverá ser encontrado em 95 dos casos. Nós já esperávamos que as habilidades fossem reconhecidas por mais da metade dos participantes da pesquisa, uma vez que habilidades associadas ao PC podem ser vistas como competências triviais às atividades de um profissional da computação, independente de sua área de atuação.

Tabela 2: Testes de proporção para habilidades relacionadas à QP3

\begin{tabular}{|l|l|l|l|}
\hline Habilidade & $\boldsymbol{p}$-value & $\begin{array}{l}\text { Intervalo de confiança } \\
\text { (menor) }\end{array}$ & $\begin{array}{l}\text { Intervalo de confiança } \\
\text { (maior) }\end{array}$ \\
\hline Coleta de dados & $8.176 \mathrm{e}-05$ & 0.63 & 0.85 \\
\hline Análise de dados & 0.0002364 & 0.62 & 0.84 \\
\hline Representação de dados & 0.003868 & 0.56 & 0.79 \\
\hline Decomposição & $2.925 \mathrm{e}-10$ & 0.81 & 0.96 \\
\hline Algoritmo & $3.49 \mathrm{e}-08$ & 0.75 & 0.93 \\
\hline Abstração & $8.176 \mathrm{e}-05$ & 0.63 & 0.85 \\
\hline Simulação & 0.4308 & 0.42 & 0.67 \\
\hline Automação & $3.49 \mathrm{e}-08$ & 0.75 & 0.93 \\
\hline Paralelização & 0.1151 & 0.47 & 0.72 \\
\hline
\end{tabular}

Por outro lado, os intervalos de confiança nos mostram que simulação e paralelismo possuem variância entre $42 \%$ e $67 \%$ e entre $47 \%$ e $72 \%$, respectivamente. Tal resultado fugiu da nossa expectativa e levantamos a questão se existe algum motivo específico para essas competências não acompanharem o mesmo padrão de resposta das demais habilidades pesquisadas. Nossas suposições para esses resultados são: (i) não houve compreensão adequada da descrição da habilidade; (ii) não houve assimilação direta da habilidade à atividade laboral, (iii) são habilidades mais restritas a um perfil de profissional, como os desenvolvedores. Essas suposições carecem de maiores investigações em pesquisas futuras.

Tabela 3: Testes de proporção para habilidades relacionadas à QP4

\begin{tabular}{|l|l|l|l|}
\hline Habilidade & $\boldsymbol{p}$-value & $\begin{array}{l}\text { Intervalo de confiança } \\
\text { (menor) }\end{array}$ & $\begin{array}{l}\text { Intervalo de confiança } \\
\text { (maior) }\end{array}$ \\
\hline Coleta de dados & 0.1892 & 0.45 & 0.70 \\
\hline Análise de dados & 0.5994 & 0.40 & 0.66 \\
\hline Representação de dados & 0.0181 & 0.23 & 0.47 \\
\hline Decomposição & 0.003868 & 0.56 & 0.79 \\
\hline Algoritmo & 0.1151 & 0.47 & 0.72 \\
\hline Abstração & 0.7928 & 0.39 & 0.64 \\
\hline Simulação & $2.648 \mathrm{e}-05$ & 0.13 & 0.35 \\
\hline Automação & 0.001625 & 0.57 & 0.81 \\
\hline Paralelização & 0.4308 & 0.42 & 0.68 \\
\hline
\end{tabular}

Continuando a análise, nós aplicamos o mesmo teste de proporção para as 


\section{CBIE-LACLO 2015}

Anais dos Workshops do IV Congresso Brasileiro de Informática na Educação (CBIE 2015)

respostas relacionadas à percepção das habilidades nas atividades cotidianas (Tabela 3). Assim, retornamos a questão de pesquisa 4: QP4 - Os profissionais da computação reconhecem que usam as habilidades associadas ao PC nas suas atividades cotidianas, fora do ambiente de trabalho?

Observando os resultados calculados na Tabela 3 vemos que decomposição e automação apresentam intervalos de confiança sempre acima de 56\%. Para as habilidades de coleta de dados, análise de dados, algoritmo, abstração e paralelização os intervalos de confianças estão em torno de 50\%. Já para as habilidades de representação de dados e simulação, os intervalos de confiança estão ambos abaixo de $50 \%$, entre $23 \%$ e $47 \%$ e entre $13 \%$ e $35 \%$, respectivamente.

Quando comparamos a percepção das habilidades nas atividades laborais e atividades cotidianas, notamos que há diferença nas proporções, conforme indica a Figura 1 e Tabelas 2 e 3. Nas atividades laborais, as habilidades coleta de dados, análise de dados, representação de dados, decomposição, algoritmo, abstração e automação são exploradas por mais da metade dos participantes da pesquisa. Já nas atividades cotidianas, apenas decomposição e automação possuem o mesmo destaque. Nossas suposições preliminares para essas diferenças são: (i) as pessoas não costumam associar habilidades exploradas no trabalho com as atividades fora do ambiente de trabalho, ou seja, tendem a separar os contextos; (ii) as pessoas não exploram essas habilidades conscientemente no seu dia a dia.

No caso das habilidades decomposição e automação, as quais tiveram destaque nas atividades cotidianas, nossas suposições para esse resultado são: (i) as pessoas reconhecem que não conseguem resolver um problema ou uma tarefa complexa em um único momento, é necessário dividir em tarefas menores para resolvê-las, ou seja, decompor; (ii) devido à variedade de recursos eletroeletrônicos, é uma atitude natural automatizar atividades para facilitar a resolver problemas ou tarefas do dia a dia.

\subsection{Ameaças à validade}

Nossa pesquisa apresenta ameaças à validade de conclusão, pois há baixo poder estatístico devido ao tamanho da amostra. Temos também ameaças à validade interna, pois muitos dos sujeitos aos quais o questionário foi enviado não responderam. Aos que responderam, podem não ter compreendido ou dado atenção suficiente a cada item do questionário ou ainda não responderam com sinceridade.

Temos ameaças à validade de construção com relação ao conceito e definição do PC e das habilidades selecionadas para a pesquisa, pois há várias definições na literatura. Temos ameaças à validade com relação às perguntas elaboradas, haja vista que elas podem não capturar os efeitos que se deseja medir. E temos ameaças à validade externa com relação à interação de seleção e tratamento, uma vez que há limitações com a representatividade da população e o método amostral.

\section{Considerações finais}

Nesse trabalho, realizamos um survey com profissionais da computação os quais atuam na academia e na indústria, para capturar a compreensão que esses profissionais têm de Pensamento Computacional em suas atividades laborais e cotidianas. Nossos resultados 


\section{CBIE-LACLO 2015}

Anais dos Workshops do IV Congresso Brasileiro de Informática na Educação (CBIE 2015)

apontam que $64 \%$ dos pesquisados desconhecem o PC e sugerem que o termo PC é pouco conhecido e compreendido pelos profissionais, tanto na indústria quanto na academia. $\mathrm{O}$ desconhecimento vai da definição do termo e da área de conhecimento às competências relevantes. Quanto as habilidades relevantes, nossos resultados sugerem que decomposição e automação são as habilidades apontadas com maior frequência tanto no contexto de atividades laborais como no cotidiano.

Ressaltamos que os resultados encontrados não podem ser generalizados devido à baixa representatividade da população e ao método não-probabilístico como selecionamos a amostra. Mesmo com essas e outras limitações já listadas, apresentamos uma iniciativa que discutiu como encontra-se o estado atual da percepção do PC entre os profissionais da computação, tanto os que atuam na academia e como na indústria.

Como trabalhos futuros, pretendemos aumentar o tamanho da amostra e mudar o método de seleção no intuito de realizar um comparativo com esse resultado inicial. Propomos também investigar de que maneira as habilidades são exploradas nas atividades cotidianas, bem como o motivo de algumas serem mais citadas que outras.

\section{Referências}

Barr V. e Stephenson, C. (2011) "Bringing computational thinking to K-12: what is Involved and what is the role of the computer science education community?". In: ACM Inroads 2. p.48-54.

$\mathrm{Hu}, \mathrm{C}$. (2011) "Computational thinking: what it might mean and what we might do about it." In: Proceedings of the 16th annual joint conference on Innovation and technology in computer science education (ITiCSE '11). ACM, New York, USA. p. 223-227.

Li, T. e Wang, T. (2012) "A Unified Approach to Teach Computational Thinking for First Year Non-CS Majors in an Introductory Course”. In: International Conference on Future Computer Supported Education, Elsevier. p. 498-503.

Mannila, L., Dagiene, V., Demo, B., Grgurin, N. Mirolo, C. Rolandsson, L. e Settle, A. (2014) "Computational Thinking in K-9 Education." In: Proceedings of the Working Group Reports of the 2014 on Innovation \& Technology in Computer Science Education Conference (ITiCSE-WGR '14), Alison Clear Clear and Raymond Lister (Eds.). ACM, New York, USA. p. 1-29.

Pokorny, K. e White, N. (2012) "Computational thinking outreach: reaching across the K-12 curriculum”. In: Journal of Computing Sciences in Colleges. 27, 5. p. 234-242.

Selby, C. e Woollard, J. (2013) "Computational thinking: the developing definition". In: Special Interest Group on Computer Science Education (SIGCSE) 2014, Atlanta, US.

Walden, J., Doyle, M., Garns, R. e Hart, Z. (2013) "An informatics perspective on computational thinking". In Proceedings of the 18th ACM conference on Innovation and technology in computer science education (ITiCSE '13). ACM, New York, USA. p. 4-9.

Wing, J. 2006. Computational thinking. Commun. ACM, 49. p.33-35. 